\title{
BMJ Open Interventions for mental health problems in children and adults with severe intellectual disabilities: a systematic review
}

\author{
Leen Vereenooghe, ${ }^{1}$ Samantha Flynn, ${ }^{2}$ Richard P Hastings, ${ }^{2,3}$ Dawn Adams, ${ }^{4}$ \\ Umesh Chauhan, ${ }^{5}$ Sally-Ann Cooper, ${ }^{6}$ Nick Gore, ${ }^{7}$ Chris Hatton, ${ }^{8}$ Kerry Hood, ${ }^{9}$ \\ Andrew Jahoda, ${ }^{6}$ Peter E Langdon, ${ }^{7}$ Rachel McNamara, ${ }^{9}$ Chris Oliver, ${ }^{10}$ \\ Ashok Roy, ${ }^{11}$ Vasiliki Totsika, ${ }^{3,12}$ Jane Waite ${ }^{13}$
}

To cite: Vereenooghe L, Flynn S, Hastings RP, et al. Interventions for mental health problems in children and adults with severe intellectual disabilities: a systematic review. BMJ Open 2018;8:e21911. doi:10.1136/ bmjopen-2018-021911

- Prepublication history and additional material for this paper are available online. To view these files, please visit the journal online (http://dx.doi. org/10.1136/bmjopen-2018021911).

Received 26 January 2018 Revised 23 April 2018 Accepted 15 May 2018

Check for updates

For numbered affiliations see end of article.

Correspondence to Dr Leen Vereenooghe; leen.vereenooghe@unibielefeld.de

\section{ABSTRACT}

Objective Mental health problems are more prevalent in people with than without intellectual disabilities, yet treatment options have received little attention. The aim of this study was to identify and evaluate the effectiveness of pharmacological and psychological interventions in the treatment of mental health problems in children and adults with severe and profound intellectual disabilities, given their difficulties in accessing standard mental health interventions, particularly talking therapies, and difficulties reporting drug side effects.

Design A systematic review using electronic searches of PsycINF0, PsycTESTS, EMBASE, MEDLINE, CINAHL, ERIC, ASSIA, Science Citation Index, Social Science Citation Index and CENTRAL was conducted to identify eligible intervention studies. Study selection, data extraction and quality appraisal were performed by two independent reviewers.

Participants Study samples included at least $70 \%$ children and/or adults with severe or profound intellectual disabilities or reported the outcomes of this subpopulation separate from participants with other levels of intellectual disabilities.

Interventions Eligible intervention studies evaluated a psychological or pharmacological intervention using a control condition or pre-post design.

Outcomes Symptom severity, frequency or other quantitative dimension (e.g., impact), as assessed with standardised measures of mental health problems.

Results We retrieved 41232 records, reviewed 573 fulltext articles and identified five studies eligible for inclusion: three studies evaluating pharmacological interventions, and two studies evaluating psychological interventions. Study designs ranged from double-blind placebo controlled crossover trials to single-case experimental reversal designs. Quality appraisals of this very limited literature base revealed good experimental control, poor reporting standards and a lack of follow-up data.

Conclusions Mental ill health requires vigorous treatment, yet the current evidence base is too limited to identify with precision effective treatments specifically for children or adults with severe and profound intellectual disabilities. Clinicians therefore must work on the basis of general population evidence, while researchers work
Strengths and limitations of this study

- To our knowledge, this is the first systematic review focused on interventions to improve the mental health of both children and adults with severe and profound intellectual disabilities.

- Review eligibility was not restricted to randomised controlled trials which limits the strength of the review's findings.

- The body of evidence we identified was very slim and does not allow for generalisation of findings for either psychological or pharmacological interventions.

to generate more precise evidence for people with severe and profound intellectual disabilities.

PROSPERO registration number CRD 42015024469.

\section{INTRODUCTION}

Intellectual disabilities affect approximately $1 \%$ of the population and are characterised by significantly impaired intellectual and adaptive skills with onset before adulthood. Their prevalence of mental health problems has been reported to be more than seven times higher than for the general population. ${ }^{1}$ People with severe and profound intellectual disabilities, as indicated by an IQ of less than 40, have limitations in problem-solving skills, cognitive and communication skills which can affect their ability to cope with stressful life events. The life circumstances of people with an intellectual disability may increase their risk of developing mental health problems or experiencing mental distress. Factors that have been identified as protective in adults without intellectual disabilities, such as employment opportunities, meaningful day activities and socially supportive networks, may be less likely to be present for people 
with intellectual disabilities and with additional impact for those with severe and profound intellectual disabilities compared with those with mild or moderate intellectual disabilities. ${ }^{2-4}$ Genetic factors may further increase the vulnerability of some people with intellectual disabilities for mental health problems, as evidenced by significant comorbidity rates of anxiety problems and psychosis in people with intellectual disabilities and certain genetic syndromes. ${ }^{5-9}$

Mental health problems are as common in people with severe and profound intellectual disabilities as in people with mild or moderate intellectual disabilities, reported to have a point prevalence of $22.4 \% .{ }^{10-14}$ Their treatment of mental health problems requires particular attention for three main reasons. First, longitudinal research investigating the mental health of children and young people with intellectual disabilities over a 14-year period suggests recovery may be poorer for those with severe intellectual disabilities, and therefore standard treatments may be suboptimal. ${ }^{10-12}$ Second, given their limitations in communication skills and understanding, people with severe and profound intellectual disabilities cannot be assumed to find talking therapies such as cognitive-behavioural therapy (CBT)-based interventions as accessible as other people do; yet these therapies are considered first-line treatments of choice for many types of mental health problems. Third, it is possible that people with intellectual disabilities are more sensitive to the side effects of pharmacotherapies, or have greater difficulties in reporting side effects when these occur, so raising the potential of more serious consequences, and the need for different dosing regimens compared with other people. The high prevalence and potentially persistent mental health problems experienced by people with severe and profound intellectual disabilities thus call for effective interventions to treat such problems and to promote well-being.

Existing systematic reviews have evaluated either the psychological or pharmacological treatment of mental health problems in people with intellectual disabilities. CBTs were found to have moderate positive treatment effects for people with intellectual disabilities who experience anger problems, anxiety and depression, ${ }^{15-17}$ but these findings are limited to adults with mild to moderate intellectual disabilities, however, as children or individuals with severe and profound intellectual disabilities were not represented in the primary studies. Reviews of pharmacological interventions have largely focused on behaviour problems independent of their association with mental health problems. For example, potentially effective interventions for behaviour problems in adults with intellectual disabilities include risperidone, lithium and antiepileptic mood stabilisers. ${ }^{18}{ }^{19}$ However, the methodological quality of the evidence and registered adverse effects indicate that the use of these pharmacological agents requires caution. ${ }^{18} 19$ While behaviour problems can be associated with mental health problems and take on a precipitating or perpetuating role, they are more indicative of emotional dysregulation than of psychiatric symptomatology, and have been demonstrated in robust studies to be distinct from other types of mental health problems. ${ }^{20}$ We have not identified reviews on treatment response and side effects to pharmacotherapies for other types of mental health problems experienced by people with severe and profound intellectual disabilities. The objective of the present systematic review was to evaluate the effectiveness of psychological and pharmacological treatments for mental health problems and their key symptoms in both children and adults with severe or profound intellectual disabilities.

\section{METHODS}

The review was conducted and written in accordance with the Preferred Reporting Items for Systematic Reviews and Meta-Analyses (PRISMA) statement. ${ }^{21}$ The review protocol was registered with PROSPERO, Centre for Reviews and Dissemination, under the reference number CRD 42015024469.

\section{Search strategy}

The search strategy was developed for two conjoint systematic reviews focused on the evaluation of both measures of and interventions for mental health problems in people with severe and profound intellectual disabilities. Although separate search terms were used for each systematic review, records identified through the respective searches were pooled together prior to the study eligibility screening to ensure that studies piloting an assessment as an intervention outcome measure would also be identified.

Initial systematic searches were conducted in the week of 13-17 July 2015 for the following databases: PsycINFO, PsycTESTS, EMBASE, MEDLINE, CINAHL, ERIC, ASSIA, Science Citation Index, Social Science Citation Index, and Cochrane Central Register of Controlled Trials (CENTRAL). Searches used Boolean terms to combine search strings for intellectual disabilities, mental health and psychological or pharmacological interventions. Instead of listing all potential diagnosis and treatments the search strategy included the most common diagnoses and treatments in conjunction with more general mental health descriptions. This approach could limit the initial records to be screened, whereas relevant studies could still be identified through the ancestry method which screens citing and cited articles of included studies and through contact with authors. A sample search strategy for the PsycINFO, PsycTESTS and ASSIA searches is provided in the online supplementary appendix. Full search strategies for each database can be requested from the authors.

Searches were updated in September 2017, to cover the time period from the original searches, and no new studies were identified from these searches. The updated searches followed the same search strategy and study screening protocol as the original searches. 


\section{Study eligibility criteria}

The following inclusion criteria were applied to (1) publication type, (2) study design, (3) participants, (4) interventions and (5) outcomes.

\section{Publication}

Peer-reviewed publications written in English, French, German or Dutch were eligible for review.

\section{Study design}

The following study designs were eligible for inclusion in the review: (A) randomised controlled trials, (B) controlled trials without randomisation, $(\mathrm{C})$ single-group pre-post designs, (D) case series with outcome measures reported as group mean data, (E) single-case experimental designs and (F) case-control studies. Observational and retrospective cohort studies, as well as case studies without a control condition or a return to baseline, were excluded.

\section{Participants}

To ensure that the outcome data were representative for people with severe and profound intellectual disabilities it was required that either a minimum of $70 \%$ of participants were diagnosed or reported as having severe or profound intellectual disabilities, or that data for participants with severe or profound intellectual disabilities were reported separately in the study. Although this was an arbitrary criterion, this was to ensure that a majority of people with severe or profound intellectual disabilities were in the study samples. Studies that did not provide any usable information about the level of intellectual disabilities within samples were excluded. No exclusions were applied concerning participants' age or gender or any other characteristics except for degree of intellectual disability.

\section{Intervention}

Eligible psychological interventions were delivered by a trained lay therapist or qualified professional who systematically applied interventions based on well-established psychological principles and techniques directly to the person with an intellectual disability, either individually or in a group. For pharmacological interventions, it was expected that the pharmaceutical agent was given with regular review by a qualified medical practitioner or health professional, and recognised at least in principle as a potential treatment for a mental health problem/ symptom.

\section{Outcomes}

Eligible outcomes were standardised assessments of mental disorders or their key symptoms which have a significant impact on daily functioning. However, we acknowledge that defining the mental and physical components of mental and physical disorders into mutually exclusive categories can be challenging, not in the least because certain components are symptomatic of multiple disorders and certain disorders have shown high rates of comorbidity with one another. For the purpose of this systematic review, the inclusion criteria for mental disorders and their symptoms were derived from the Diagnostic and Statistical Manual of Mental Disorders Fourth Edition (DSM-IV), ${ }^{22}$ as this version was most likely to be used by the primary studies to be identified by the systematic review. Mental and behavioural disorders, and their key symptoms, eligible for review fell within the following classifications: (A) attention-deficit and disruptive behaviour disorders, (B) tic disorders, (C) other disorders of infancy, childhood or adolescence, (D) schizophrenia and other psychotic disorders, (E) mood disorders, (F) anxiety disorders, $(\mathrm{G})$ somatoform disorders, (H) factitious disorders, (I) dissociative disorders, (J) eating disorders, (K) adjustment disorders and (L) personality disorders.

Studies focused on key symptoms of mental disorders were included as not all treatment offers a holistic approach, and interventions may instead aim to alleviate one or more symptoms of a disorder. By contrast, challenging behaviours and behaviour problems may be associated with or indicative of underlying mental disorders $^{2023}$ but are not recognised as a key diagnostic feature of the above listed mental disorders and are hence excluded from this review.

The broad scope of the systematic review in terms of study designs, type of interventions and range of participants was advised as initial scoping searches indicated that only few studies included individuals with severe and profound intellectual disabilities.

A single post hoc exclusion criterion was applied to exclude records from the searches published prior to 1980 (n=106 records, but not fully checked for inclusion criteria), coinciding with the publication of the DSM-III. ${ }^{24}$ This assured a minimal level of consistency in the recognition and diagnosis of mental health problems from DSM-III through to DSM-IV. It is likely that there would have been a delay between the publication of the DSM-III and its first use in published research, but searches back to 1980 were essential to ensure that no potentially relevant studies were missed.

\section{Study selection}

Data collection and abstract screening were performed by the first author (LV). Twenty per cent of records were also screened by the second author (SF), leading to an overall agreement rate of $99.8 \%$ and a kappa coefficient of 0.91 for studies to proceed to full-text evaluation. Second screening a proportion of results is an accepted practice when a review is large and resources are limited. ${ }^{25}$ The overall inclusion rate for the screening of titles and abstracts was 2.3\%. Full-text review of 573 articles was performed independently by the two reviewers ( $\mathrm{LV}$ and $\mathrm{SF}$ ), which resulted in a kappa coefficient of 0.76 for inclusion in the review and the data extraction stage. Eleven disagreements between the two reviewers were resolved through joint discussion. All disagreements concerned the proportion of participants with 
severe and profound intellectual disabilities and were not related to study design, intervention or outcomes. The review of one full-text article required consultation with the third author (RPH) to determine whether this study met the review eligibility criteria regarding mental health outcomes. Upon discussion, the paper was excluded from the review.

Next, reference lists and citation records of all included studies were screened to identify additional papers that may not have fulfilled the search term criteria. No additional studies were identified in this way.

\section{Data extraction and quality synthesis}

Data extraction was conducted by the second author and reviewed by the first author for variables including: study design, study population, intervention, outcome measures and follow-up data.

The certainty in the evidence for each outcome measure could not be assessed with the Grades of Recommendation, Assessment, Development and Evaluation approach, ${ }^{26-28}$ as used by the Cochrane collaboration and national guideline organisations such as National Institute for Health and Care Excellence (NICE) in the UK, due to the incomparability of identified studies in terms of study design, interventions and outcomes. Likewise, it was not possible to conduct a meta-analysis or provide other summary measures because no two studies addressed the same mental health problem using a similar intervention.

Both reviewers independently performed a critical appraisal of all included studies. No disagreements were recorded at either stage. The assessment followed the Critical Appraisal Skills Programme (CASP) ${ }^{29} 30$ Checklists or the quality indicators for within single-subjects research, ${ }^{31}$ dependent on the study design.

\section{Patient and public involvement}

Patients and public were not involved in the conception, development or implementation of this systematic review, nor in the selection of outcome measures and the interpretation of the study findings.

\section{RESULTS}

The search strategy for the conjoint systematic review identified 24883 unique records, of which 573 were retained for full-text eligibility screening. The study selection process is illustrated in figure 1. Excluded articles most commonly did not meet the eligibility criteria concerning the severity of intellectual disabilities of study participants $(n=242)$. Initial records were also excluded based on their study design $(\mathrm{n}=113)$, a publication date prior to $1980 \quad(\mathrm{n}=106)$, because the intervention or outcomes were not focused on recognised mental health

\begin{tabular}{|c|c|c|c|}
\hline $\begin{array}{l}\text { Records identified through } \\
\text { database searching } \\
n=41232\end{array}$ & \multicolumn{2}{|c|}{$\begin{array}{l}\text { Additional records identified } \\
\text { through other sources } \\
n=0\end{array}$} & \\
\hline \multicolumn{2}{|c|}{$\begin{array}{l}\text { Records screened after duplicates removed } \\
\qquad n=24883\end{array}$} & & $\begin{array}{l}\text { Records excluded } \\
\qquad n=24310\end{array}$ \\
\hline \multicolumn{4}{|l|}{$\downarrow$} \\
\hline \multicolumn{2}{|c|}{$\begin{array}{l}\text { Full-text articles assessed for eligibility } \\
\qquad n=573\end{array}$} & \multirow{5}{*}{\multicolumn{2}{|c|}{$\begin{array}{l}\text { Full-text articles excluded } \\
\text { - Published prior to } 1980: \mathrm{n}=106 \\
\text { - No participants with severe ID: } \mathrm{n}=141 \\
\text { - Insufficient proportion of participants } \\
\text { with severe ID or no separate reporting } \\
\text { of outcome data: } \mathrm{n}=101 \\
\text { - Conference abstract: } \mathrm{n}=38 \\
\text { - No quantitative mental health } \\
\text { outcome measure: } \mathrm{n}=59 \\
\text { - Non-eligible language of publication: } \mathrm{n}=4 \\
\text { - Full-length paper not obtained: } \mathrm{n}=6 \\
\text { - Non-eligible study design: } \mathrm{n}=113\end{array}$}} \\
\hline \multicolumn{2}{|l|}{$\downarrow$} & & \\
\hline \multicolumn{2}{|c|}{$\begin{array}{l}\text { Studies included in qualitative synthesis } \\
\qquad n=5\end{array}$} & & \\
\hline \multicolumn{2}{|l|}{$\downarrow$} & & \\
\hline \multicolumn{2}{|c|}{$\begin{array}{l}\text { Additional studies identified } \\
\text { through ancestry method and author contact } \\
n=0\end{array}$} & & \\
\hline
\end{tabular}

\section{Studies included in qualitative synthesis} $n=5$

Figure 1 Preferred Reporting Items for Systematic Reviews and Meta-Analyses (PRISMA) flow diagram. ID, intellectual disability. 
problems $(n=59)$, due to their publication status (e.g., conference abstracts; $n=38$ ), or because the full-text paper could either not be retrieved $(n=6)$ or was published in a non-eligible language $(n=4)$. In total, five studies were included in the review and are described in table 1 . Three studies included only adults with intellectual disabilities: a double-blind placebo controlled crossover trial ${ }^{32}$ and a single-case experimental reversal design of pharmacotherapy, ${ }^{33}$ as well as a single-case experimental reversal design of a psychological intervention. ${ }^{34}$ Two studies included children and young people: a randomised trial of pharmacotherapy by White and Aman ${ }^{35}$ and a singlecase study of a psychological intervention for a 13-year-old girl. ${ }^{36}$

\section{Psychological interventions}

Two studies evaluated interventions based on psychological principles. Interventions were offered for symptoms of depressive disorder, and to manage tic frequency in Gilles de la Tourette syndrome.

In a single-case experimental $\mathrm{ABAB}$ design, Lindauer and colleagues $^{34}$ offered an enriched environment for the management of major depressive disorder in a 23-year-old woman with severe intellectual disabilities who also presented with self-injurious behaviour. Pre-existing treatment of the mood disorder with carbamazepine $(5.3 \mathrm{mg} / \mathrm{kg} /$ day $)$ was continued during the study. The enriched environment setting was a $3 \mathrm{~m} \times 3 \mathrm{~m}$ padded room, in an inpatient unit, in which stimuli were present that were chosen following a paired-choice assessment to identify the woman's preferred stimuli and assess signs of positive and negative affects. Smiling, giggling and laughing were considered examples of positive 'affect', whereas frowning, whining, crying and verbal expressions such as 'I am sad' were identified as signs of negative 'affect'. No other outcome measures relating to the mood disorder were employed. Behavioural observations, through a one-way mirror, showed that the enriched environment increased signs of positive affect and decreased signs of negative affect, in particular during the second intervention phase. The lack of follow-up measures and the delivery of interventions in a padded room in an inpatient setting reduce the ecological validity of this intervention. Likewise, the replicability of findings is impeded in terms of participant selection and intervention fidelity (see table 2).

Zarkowska ${ }^{36}$ adopted a basic single-case experimental design to examine interventions for vocal and motor tics in a 13-year-old girl with Gilles de la Tourette syndrome and severe intellectual disabilities. Two treatment probes, cued relaxation and interruption, were evaluated using an $\mathrm{ABA}$ return to baseline design for each intervention comprised of a $5 \mathrm{~min}$ baseline recording, a $5 \mathrm{~min}$ intervention and a $5 \mathrm{~min}$ postbaseline recording. Cued relaxation appeared to lead to better outcomes but neither intervention had lasting effects and interruption increased vocal tic frequency. The study design showed strong external and social validity and provided clear descriptions of dependent and independent variables (see table 2). However, internal validity was weak and the ABA design was not the most suitable for demonstrating experimental control. Following the evaluation of treatment probes, the study continued as an A-B case study implementing successive interventions of relaxation training, treatment with clonidine and treatment with pimozide. Due to the non-controlled nature of these interventions, their respective outcome data and follow-up data were not considered eligible for inclusion in this review.

The replicability of findings from both studies is hindered by a lack of information regarding participant selection, physical setting of the intervention, implementation fidelity and the reliability of outcome measurements.

\section{Pharmacological interventions}

Two double-blind placebo controlled crossover trials and one single-case experimental reversal design evaluated pharmacological interventions for use in people with severe intellectual disabilities and mental health problems.

Aman and colleagues ${ }^{32}$ employed within-group randomisation of order of administration of 4-week treatment with imipramine, in a dosage of $3 \mathrm{mg} / \mathrm{kg} /$ day, and 4 weeks with placebo, with 1-week drug-free in between. Interventions were offered to five adults with severe intellectual disabilities and depressive symptoms, in addition to a group of five adults with acting-out behaviours. The latter were not eligible for inclusion in this review as these behaviours were not considered a mental health problem. Eligible depressive symptoms were based on evidence from prior research studies and required behavioural observation instead of information obtained from diagnostic interviews. Symptoms included 'seclusion and social withdrawal, sleep loss, weight loss, tearfulness or the appearance of sad affect, and a pervasive lack of overt

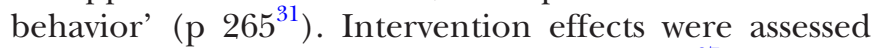
with the Aberrant Behavior Checklist $(\mathrm{ABC})^{37}$ and indicated imipramine to have a detrimental effect on symptoms related to irritability, lethargy and hyperactivity, and no effect on stereotypical behaviours and inappropriate speech. Adverse effects were recorded but not described separately for the five adults with severe intellectual disabilities and depressive symptoms. For one person with affective symptoms, imipramine was found to improve behaviour and relieve chronic constipation.

White and $\mathrm{Aman}^{35}$ evaluated the use of pimozide on maladaptive behaviours and hyperactivity, in young people and adults with moderate to profound intellectual disabilities. Following a 4-week baseline, the eight participants received two 4-week treatments with either pimozide, in a dosage of $0.12 \mathrm{mg} / \mathrm{kg} /$ day, or placebo, with a 1-week washout period between intervention phases. Treatment effects were evaluated using assessments with the $\mathrm{ABC}$ for the last 3 weeks of each intervention. Hyperactivity scores on the ABC reduced following the intervention, as did irritability levels, based on nurses' behaviour ratings 


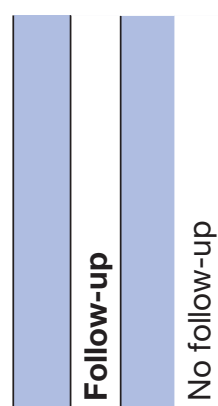

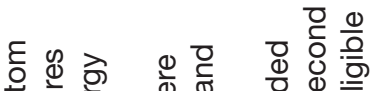

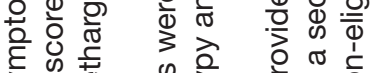

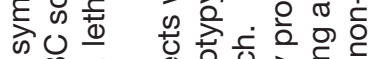
0 के

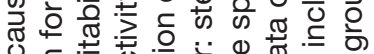

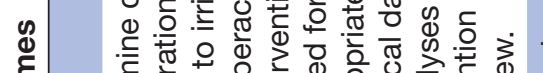

है

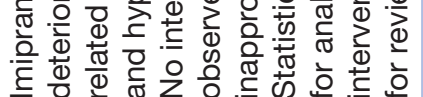

วิ

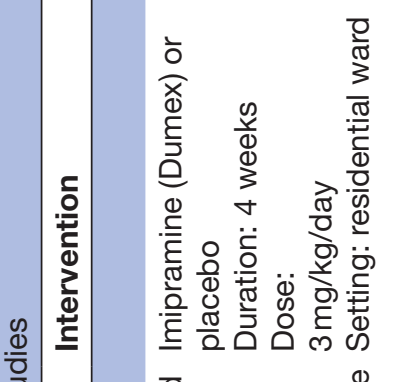

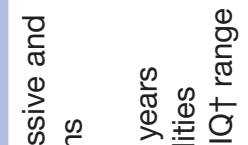

कू

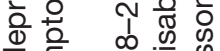

है हा

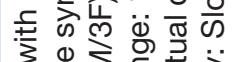

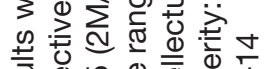

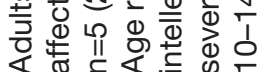

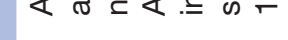
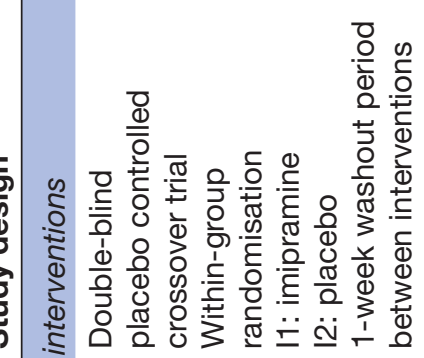

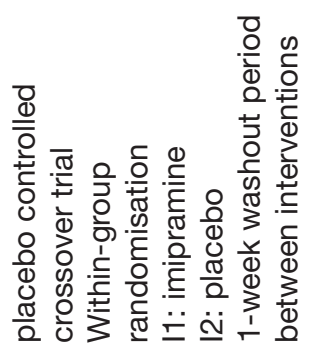
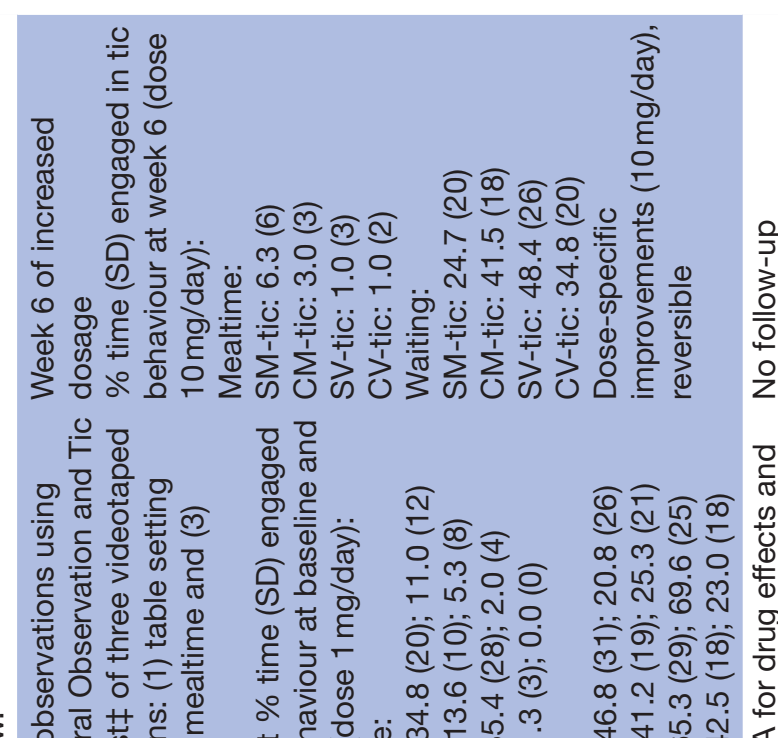

ఫृ 으

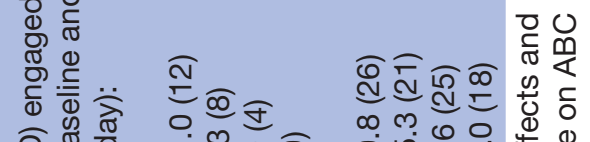
की ब † जo

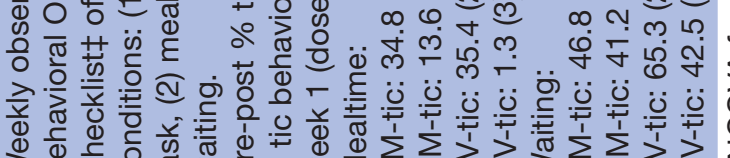
ஸे

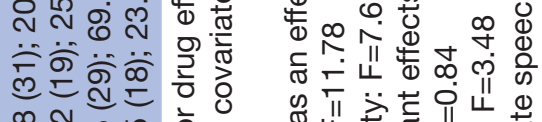

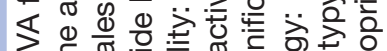

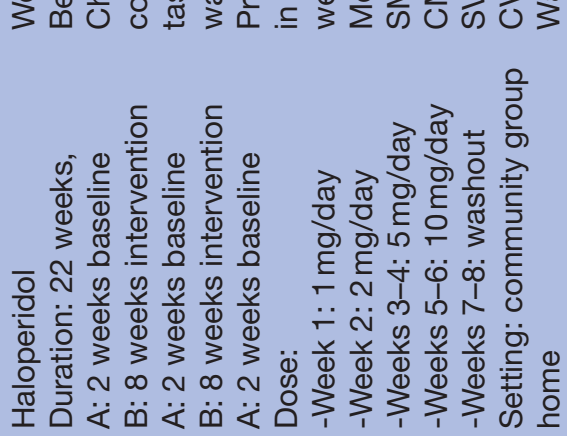

$\underline{\sigma}$
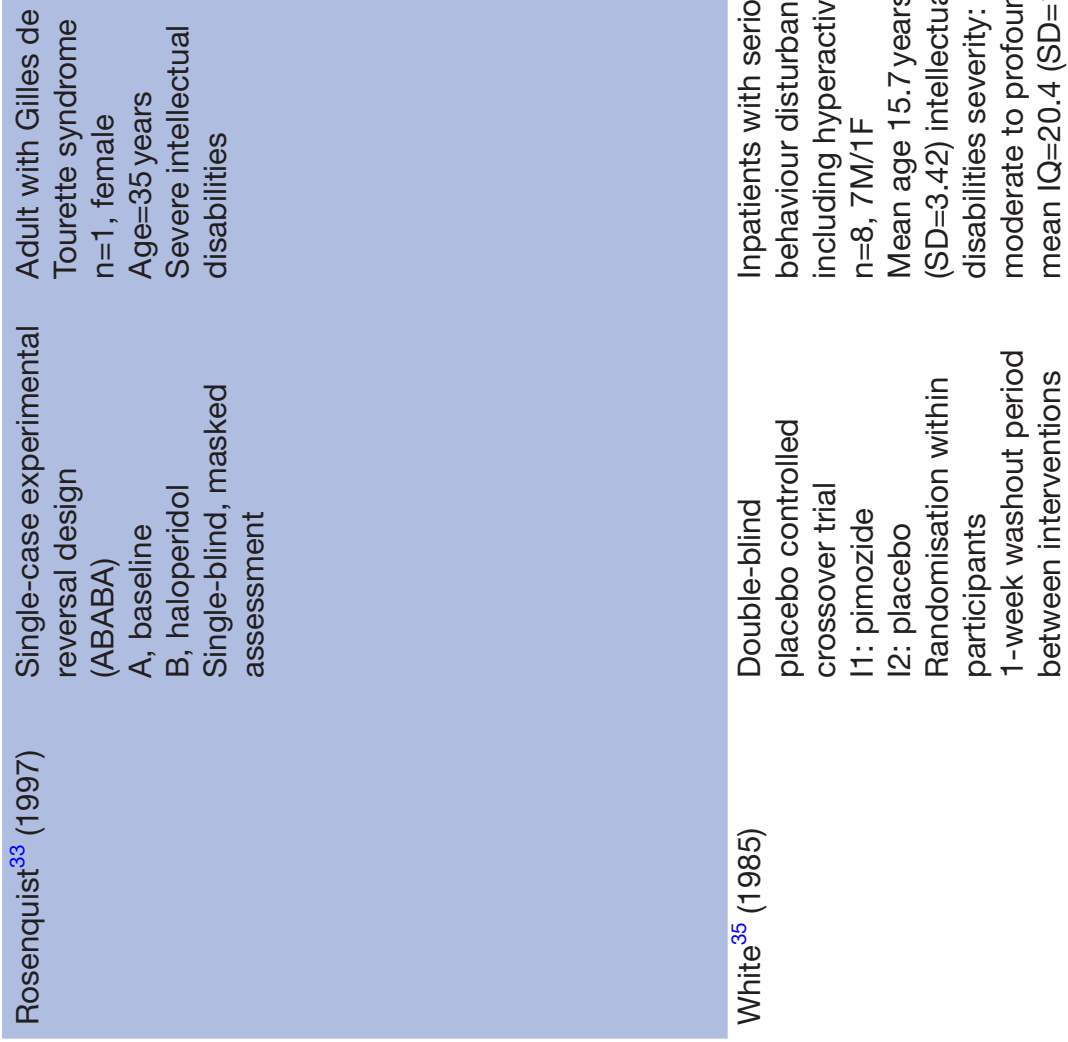

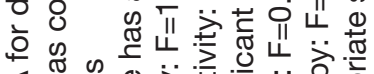
ठ․ㅠ 0

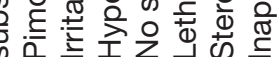
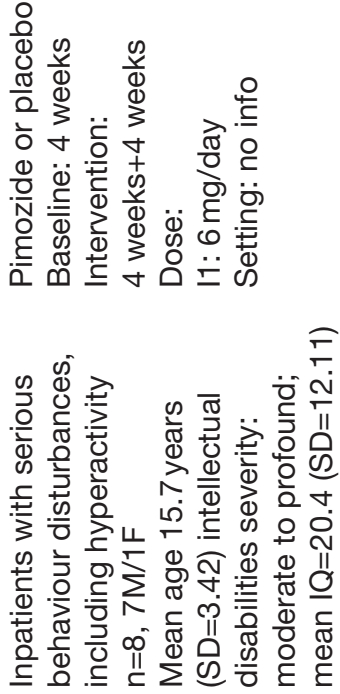

\section{$\underline{E}$}

TI

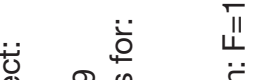

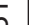
$\frac{2}{\frac{2}{2}}$

$\sqrt{\infty}$

: 


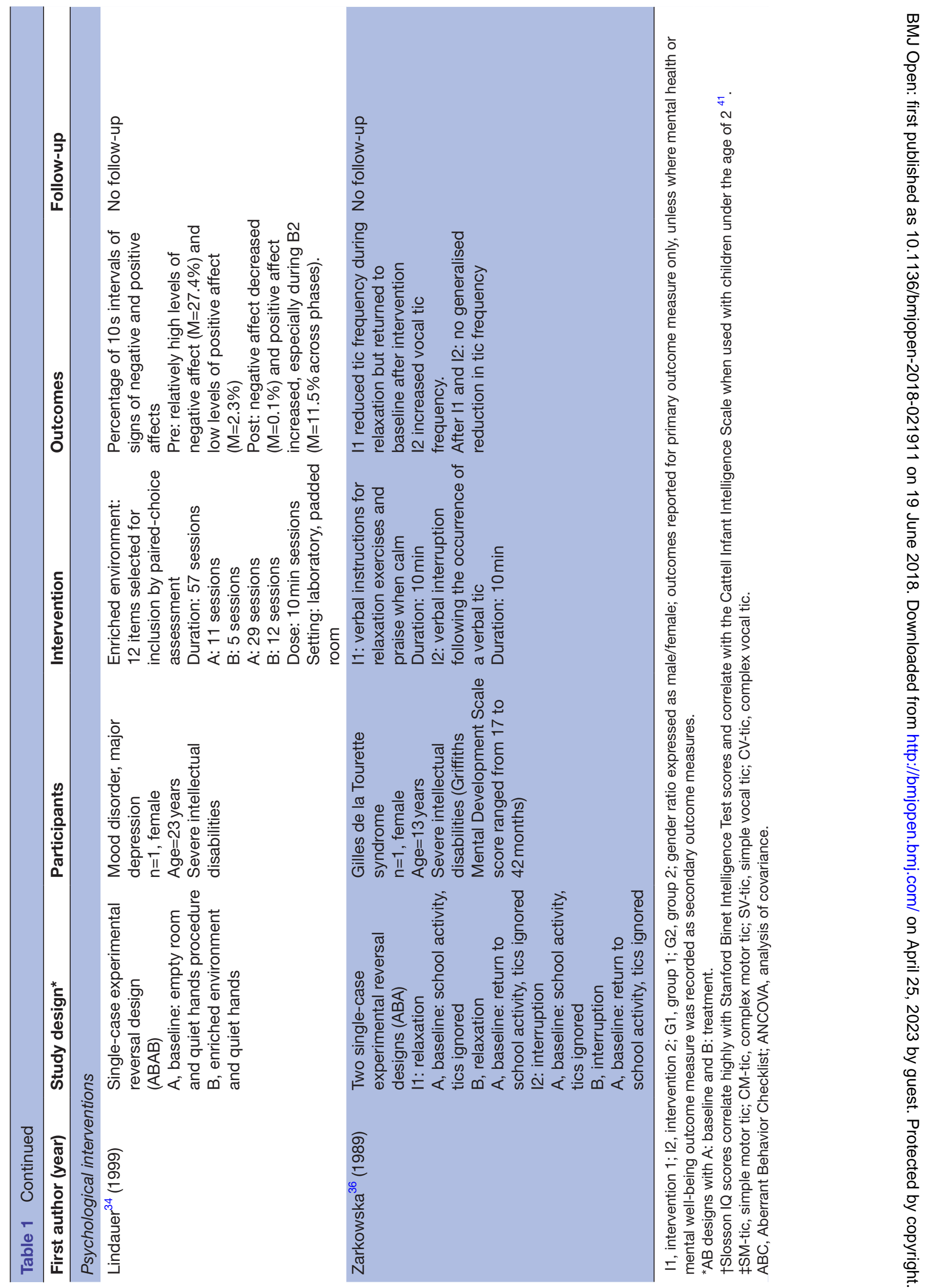


Table 2 Quality appraisal of single-subject studies using the Quality Indicators within Single-Subject Research ${ }^{31}$

\begin{tabular}{|c|c|c|c|}
\hline Quality indicator & Lindauer et $a l^{34}$ & Rosenquist et al ${ }^{33}$ & Zarkowska $^{36}$ \\
\hline \multicolumn{4}{|l|}{ Participant description and setting } \\
\hline $\begin{array}{l}\text { Ability to select individuals with similar } \\
\text { characteristics }\end{array}$ & Yes & Yes & Yes \\
\hline $\begin{array}{l}\text { Replicability of participant selection } \\
\text { process }\end{array}$ & No & No & No \\
\hline Replicability of physical setting & Yes & Yes & Partial \\
\hline \multicolumn{4}{|l|}{ Dependent variable } \\
\hline Described with operational precision & Yes & Yes & Yes \\
\hline Measured to generate a quantifiable index & Yes & Yes & Yes \\
\hline Measure is valid and replicable & Yes & Yes & Yes \\
\hline Measurements repeated over time & Yes & Yes & No \\
\hline $\begin{array}{l}\text { Measures assessed in terms of reliability or } \\
\text { interobserver agreement }\end{array}$ & Yes & Yes & No \\
\hline \multicolumn{4}{|l|}{ Independent variable } \\
\hline Described with replicable precision & Yes & Yes & Yes \\
\hline $\begin{array}{l}\text { Systematically manipulated and under } \\
\text { control of experimenter }\end{array}$ & Yes & Yes & Yes \\
\hline Overt measure of implementation fidelity & No & Not applicable & No \\
\hline \multicolumn{4}{|l|}{ Baseline } \\
\hline Repeated measurements baseline & Yes & Yes & No \\
\hline Described with replicable precision & Yes & Yes & Yes \\
\hline \multicolumn{4}{|l|}{ Experimental control/internal validity } \\
\hline $\begin{array}{l}\text { Minimum of three demonstrations of } \\
\text { experimental effect at three points in time }\end{array}$ & Yes & Yes & No \\
\hline Controlling for threats to internal validity & Unclear & Yes & Unclear \\
\hline Document a pattern of experimental control & Yes & Yes & Yes \\
\hline \multicolumn{4}{|l|}{ External validity } \\
\hline $\begin{array}{l}\text { Effects replicated across participants, } \\
\text { settings or materials }\end{array}$ & Yes & Yes & No \\
\hline \multicolumn{4}{|l|}{ Social validity } \\
\hline Dependent variable is socially important & Yes & Yes & Yes \\
\hline Magnitude of change is socially important & Yes & Yes & Yes \\
\hline $\begin{array}{l}\text { Implementation of independent variable is } \\
\text { practical and cost-effective }\end{array}$ & Yes & Yes & Yes \\
\hline $\begin{array}{l}\text { Implementation of independent variable } \\
\text { over extended period of time, by typical } \\
\text { intervention agents and in typical contexts }\end{array}$ & Yes & Yes & Yes \\
\hline
\end{tabular}

of participants. No intervention effects were observed for ABC lethargy, stereotypy and inappropriate speech domains. Furthermore, behavioural observations also did not identify any treatment effects.

The methodological quality of these two studies was confirmed using the CASP quality appraisal checklist (see table 3). However, follow-up measures were notably absent and sample sizes too small to provide sufficient power for the conducted statistical analyses. Additionally, the period of treatment was of too short duration, as imipramine can take up to 6 weeks to be effective in the general population, so that intervention was of poor design.

The only fully experimental single subject experimental design study evaluated the effect of haloperidol on tic frequency in a 35-year-old woman with Gilles de la Tourette syndrome and severe intellectual disabilities. ${ }^{33}$ Using an ABABA design, the dose of haloperidol was gradually increased during the intervention phases and maximal effectiveness was reached with the highest dosage of $10 \mathrm{mg} /$ day. Weekly behavioural observation at the community residential setting where the participant 
Table 3 Critical Appraisal Skills Programme (CASP Checklists) ${ }^{40}$ for studies with $n>1$

\begin{tabular}{|c|c|c|}
\hline Quality indicator & Aman et $a l^{32 \star}$ & White and Aman ${ }^{35 *}$ \\
\hline \multicolumn{3}{|l|}{ Validity of the results } \\
\hline Study addresses a clearly focused issue & Yes & Yes \\
\hline $\begin{array}{l}\text { Exposure accurately measured to } \\
\text { minimise bias }\end{array}$ & Yes & Yes \\
\hline $\begin{array}{l}\text { Identification of all important } \\
\text { confounding factors }\end{array}$ & Yes & Yes \\
\hline $\begin{array}{l}\text { Design and/or analysis account for } \\
\text { confounding factors }\end{array}$ & $\begin{array}{l}\text { No: length of intervention too short to observe } \\
\text { treatment effects }\end{array}$ & $\begin{array}{l}\text { No: length of intervention too short } \\
\text { to observe treatment effects }\end{array}$ \\
\hline Description of study results & Yes & Yes \\
\hline Precision of study results & $\begin{array}{l}\text { No exact } p \text { values, no effect sizes, no } \\
\text { differentiation between depressive-like and } \\
\text { acting-out group }\end{array}$ & No exact $p$ values, no effect sizes \\
\hline Believability of study results & Yes & Yes \\
\hline \multicolumn{3}{|l|}{ Impact of the results } \\
\hline Results applicable to local population & Yes & Yes \\
\hline Results in line with available evidence & No & Yes \\
\hline Implications for practice & $\begin{array}{l}\text { Length of intervention too short to draw } \\
\text { conclusions regarding implications }\end{array}$ & $\begin{array}{l}\text { The study is now outdated given } \\
\text { improved knowledge on the risks of } \\
\text { the long-term use of the drug }\end{array}$ \\
\hline
\end{tabular}

${ }^{*}$ CASP Checklists for randomised controlled trials.

lived showed reduced tic frequencies during mealtimes, nearing zero levels, and during waiting times. Intervention effects reversed when the dose was lowered. These findings are considered reliable due to masked assessment and reversal design, alongside the replicability of measures and intervention, see table 2.

\section{Overall quality appraisal of the evidence base}

Methodological quality of the identified studies was poor, with concern in terms of small sample sizes, lack of masked assessment and lack of follow-up measures. By contrast, reporting standards were generally high in terms of variable descriptions and the internal and external validity of the results. Implications of the quality appraisal are integrated in the study descriptions above, whereas a detailed overview of the quality review for each study is reported in tables 2 and 3 .

\section{DISCUSSION}

Despite their very high rates of mental health problems, there is a lack of research in interventions that explicitly target mental health problems in people with severe and profound intellectual disabilities. The scope of this review was wide. However, only five studies were eligible for inclusion and the findings are inconclusive at best. This is highly problematic for clinicians who have to manage these disorders and can only rely on the use of interventions designed for the general population, despite the likely limitations and inaccessibility of these for people with severe intellectual disabilities.

Haloperidol was demonstrated to improve tics, but in a single person. Pimozide was reported to reduce hyperactivity and other behaviour problems, ${ }^{35}$ but it is not a recognised treatment for hyperactivity in the general population; and NICE concludes that there is no evidence that antipsychotic drugs are of use in this condition ${ }^{38}$. The sedative properties of pimozide can calm disturbed patients in the short term, but it is not recommended for long-term use in view of potential side effects, which includes death, and its use is therefore reserved for schizophrenia only. While meeting the inclusion criteria of the review, the study is therefore outdated given subsequent advances in knowledge about this class of drugs. Imipramine caused deterioration of affective symptoms, but the study was poorly designed by today's standards, including the drug not being prescribed for long enough duration 
to be effective. ${ }^{32}$ Additionally, the use of imipramine has declined in the whole population since the introduction of selective serotonin reuptake inhibitors in the 1980s and other newer antidepressant agents, on the basis of side effect profile. Empirical evidence for current pharmacological interventions has not yet been published.

Evidence for the effectiveness of psychological interventions is also weak in the absence of controlled trials or high-quality single-case experimental designs (such as multiple baseline approaches). Across intervention types, two studies aimed to reduce tic frequency in people with severe intellectual disabilities and Gilles de la Tourette syndrome yielding putative positive effects for relaxation techniques and treatment with haloperidol. Evidence relating to common mental health problems (eg, anxiety, depression) was notably very limited. Studies including children with severe and profound intellectual disabilities involved different interventions than for studies with adults and while the geographic spread of the research was diverse, all included studies were conducted in English-speaking countries. Overall, a quantitative synthesis of the evidence was not possible due to the heterogeneity of the identified studies as no two studies addressed the same mental health problem with a similar intervention or similar outcome measures. Furthermore, the total sample size across the five identified studies was only 16 participants: nine children and seven adults, nine male and seven female. Finally, the review demonstrates that research in this area has stalled over the last decade. The most recent study we identified was published nearly two decades ago, ${ }^{34}$ while the methodologically stronger studies using controlled design employed outdated pharmacotherapies that are currently not recommended due to their potential side effects. ${ }^{36} 39$

\section{Strengths and limitations}

Strengths of this systematic review are the rigour with which it was conducted. In line with PRISMA guidelines, the prior publication of the review protocol enhances its transparency and replicability, while double reviewing of full-length articles and quality appraisal strengthens the findings. The current review improves upon previous reviews in this area by employing a broader scope to identify both psychological and pharmacological interventions for a range of mental health problems. In spite of this, our findings show that this area of research has received very little attention over the years with no recent treatment studies being identified and pharmacological interventions having employed drugs that would no longer comply with today's medical standards.

Limitations of the study relate to the search strategy. The systematic search did not include terms for every specific possible disorder or potential treatment, neither did it include a wide range of behavioural descriptions. In spite of this, we identified a considerably large number of potential records. Meanwhile, requiring at least $70 \%$ people with severe and profound intellectual disabilities to be included in a sample where outcomes are not reported separately for this group was a pragmatic decision so people with severe and profound intellectual disabilities would be sufficiently represented in the review findings. However, reducing the required proportion of participants with severe and profound intellectual disabilities to $50 \%$ would not have added any eligible studies (a post-hoc review check completed by the first author).

\section{Explanations and implications}

A major challenge in mental health research for people with severe and profound intellectual disabilities, including this systematic review, lies with the selection of study outcomes. The appropriateness of measures such as the $\mathrm{ABC}^{37}$ can be questioned when used to assess the wide spectrum of symptoms of mental health problems. However, the ABC was found to be one of the few reliable measures relating to mental health problems for individuals with severe and profound intellectual disabilities. ${ }^{40}$ Indeed, behavioural outcomes can assess key symptoms of mental disorders according to International Classification of Diseases 10th Revision criteria, but can equally be associated with distress and reduced quality of life. While this diagnostic taxonomy was practical for conducting the systematic review, it may not be sufficient to evaluate all relevant interventions aimed at improving the general well-being of people with severe and profound intellectual disabilities.

The scarcity of trials addressing the mental health needs of people with severe and profound intellectual disabilities is worrisome in light of the fact that they do experience mental health problems. Yet, there is awareness of the mental health needs in this population among researchers and clinicians as is evident from the wide range of descriptive case reports, which did not provide empirical evidence for the effectiveness of an intervention. On a positive note, the 101 studies identified as including at least some individuals with severe and profound intellectual disabilities show that this population is not routinely excluded from clinical practice evaluations. Although beyond the objectives of this systematic review, a scoping overview of the range of interventions evaluated in these studies and those being offered in routine clinical practice could help set the direction to guide future research. Establishing evidence-based interventions to treat mental health problems in people with severe and profound intellectual disabilities requires more research with stronger methodological designs.

\section{Future directions}

Challenging the status quo and developing an evidence base from which to treat people with severe and profound intellectual disabilities and mental health problems is a joint responsibility of practitioners and researchers. Bidirectional knowledge transfer is particularly important in this regard: research into severe and profound intellectual disabilities making its way into the training of practitioners, as well as practitioners highlighting difficulties in assessment and treatment that need addressing. Commissioning and exploring funding opportunities to conduct research into evidence-based pharmacological 
and psychological interventions, and an open discussion regarding the ethical considerations of research involving people who may lack the capacity to consent also require attention. A large inequality in evidence for effective treatments for mental health problems is experienced by children and adults with severe and profound intellectual disabilities. Until this inequality is adequately addressed, health services need to provide treatments found to be effective for people with mild to moderate intellectual disabilities where they exist-although the availability of interventions for this population is also poor in comparison to interventions for people without intellectual disabilities. Particular attention should be given to how these treatments might affect people with severe and profound intellectual disabilities differently regarding symptom presentation and outcome assessment. The accessibility of a range of psychological therapies should also be taken into account, as well asside effect reporting which may indicate a need for differences in dosing regimens. Keeping detailed accounts of how treatments were subsequently modified will benefit the development of a more solid evidence base.

\author{
Author affiliations \\ ${ }^{1}$ Faculty of Psychology and Sports Science, Bielefeld University, Bielefeld, Germany \\ ${ }^{2}$ Centre for Educational Development, Appraisal and Research, University of \\ Warwick, Coventry, UK \\ ${ }^{3}$ Centre for Developmental Psychiatry and Psychology, Department of Psychiatry, \\ School of Clinical Sciences at Monash Health, Monash University, Clayton, Victoria, \\ Australia \\ ${ }^{4}$ Autism Centre of Excellence, Griffith University, Brisbane, Queensland, Australia \\ ${ }^{5}$ Mackenzie Chair in Primary Care Medicine, School of Medicine, University of \\ Central Lancashire, Preston, UK \\ ${ }^{6}$ Institute of Health and Wellbeing, University of Glasgow, Glasgow, UK \\ ${ }^{7}$ Tizard Centre, University of Kent, Canterbury, UK \\ ${ }^{8}$ Faculty of Health and Medicine, Lancaster University, Lancaster, UK \\ ${ }^{9}$ Centre for Trials Research, Cardiff University, Cardiff, UK \\ ${ }^{10}$ School of Psychology, University of Birmingham, Birmingham, UK \\ ${ }^{11}$ Brooklands Hospital, Coventry and Warwickshire Partnership NHS Trust, Coventry, \\ UK \\ ${ }^{12}$ Centre for Education Studies, University of Warwick, Coventry, UK \\ ${ }^{13}$ School of Life \& Health Sciences, Aston University, Birmingham, UK
}

Acknowledgements We express our gratitude to Professor Nigel Beail, Professor Michael Kerr and Dr Howard Ring for their contributions to the development of the research proposal.

Contributors RPH, DA, UC, SAC, NG, CH, KH, AJ, PEL, RMN, CO, AR, VT and JW conceived the study and acquired funding. LV and RPH designed and registered the review protocol. LV and SF conducted the systematic searches, study selection and data collection. LV wrote the manuscript. All authors provided methodological and clinical perspectives, commented on manuscript drafts and read and approved the final version of the manuscript.

Funding This work was supported by the Baily Thomas Charitable Fund (Reference No: TRUST/RNA/AC/SG/3543/6297), and was sponsored by the University of Warwick (Reference No: REG0-2015-1605).

Competing interests None declared.

Patient consent Not required.

Provenance and peer review Not commissioned; externally peer reviewed.

Data sharing statement To obtain the full search strategies for each database please contact leen.vereenooghe@uni-bielefeld.de or s.flynn.1@warwick.ac.uk. This systematic review presents previously published data. Please refer to the original articles and their authors for these research data.
Open access This is an open access article distributed in accordance with the Creative Commons Attribution Non Commercial (CC BY-NC 4.0) license, which permits others to distribute, remix, adapt, build upon this work non-commercially, and license their derivative works on different terms, provided the original work is properly cited and the use is non-commercial. See: http://creativecommons.org/ licenses/by-nc/4.0/

(C) Article author(s) (or their employer(s) unless otherwise stated in the text of the article) 2018. All rights reserved. No commercial use is permitted unless otherwise expressly granted.

\section{REFERENCES}

1. Hughes-McCormack LA, Rydzewska E, Henderson A, et al. Prevalence of mental health conditions and relationship with general health in a whole-country population of people with intellectual disabilities compared with the general population. BJPsych Open 2017;3:243-8.

2. Deb S, Thomas M, Bright C. Mental disorder in adults with intellectual disability. 1: Prevalence of functional psychiatric illness among a community-based population aged between 16 and 64 years. J Intellect Disabil Res 2001;45:495-505.

3. Emerson E, Hatton C, Felce D, et al. Learning disabilities the fundamental facts. London: The Foundation for People with Learning Disabilities, 2001.

4. Hulbert-Williams L, Hastings RP. Life events as a risk factor for psychological problems in individuals with intellectual disabilities: a critical review. J Intellect Disabil Res 2008;52:883-95.

5. Cordeiro L, Ballinger E, Hagerman R, et al. Clinical assessment of DSM-IV anxiety disorders in fragile $X$ syndrome: prevalence and characterization. J Neurodev Disord 2011;3:57-67.

6. Richards C, Moss J, O'Farrell L, et al. Social anxiety in cornelia de lange syndrome. J Autism Dev Disord 2009;39:1155-62.

7. Hyman P, Oliver C, Hall S. Self-injurious behavior, self-restraint, and compulsive behaviors in Cornelia de Lange syndrome. Am J Ment Retard 2002;107:146-54.

8. Dykens EM. Anxiety, fears, and phobias in persons with Williams syndrome. Dev Neuropsychol 2003;23:291-316.

9. Krefft M, Frydecka D, Adamowski T, et al. From Prader-Willi syndrome to psychosis: translating parent-of-origin effects into schizophrenia research. Epigenomics 2014;6:677-88.

10. Cooper SA, Smiley E, Morrison J, et al. Mental ill-health in adults with intellectual disabilities: prevalence and associated factors. $\mathrm{Br} \mathrm{J}$ Psychiatry 2007;190:27-35.

11. Einfeld SL, Tonge BJ. Population prevalence of psychopathology in children and adolescents with intellectual disability: II. Epidemiological findings. J Intellect Disabil Res 1996;40:99-109.

12. Emerson $E$, Hatton $C$. Mental health of children and adolescents with intellectual disabilities in Britain. Br J Psychiatry 2007;191:493-9.

13. Hove O, Havik OE. Developmental level and other factors associated with symptoms of mental disorders and problem behaviour in adults with intellectual disabilities living in the community. Soc Psychiatry Psychiatr Epidemiol 2010;45:105-13.

14. Smiley E, Cooper SA, Finlayson J, et al. Incidence and predictors of mental ill-health in adults with intellectual disabilities: prospective study. Br J Psychiatry 2007;191:313-9.

15. Unwin G, Tsimopoulou I, Kroese BS, et al. Effectiveness of cognitive behavioural therapy (CBT) programmes for anxiety or depression in adults with intellectual disabilities: A review of the literature. Res Dev Disabil 2016;51-52:60-75.

16. Nicoll M, Beail N, Saxon D. Cognitive behavioural treatment for anger in adults with intellectual disabilities: a systematic review and metaanalysis. J Appl Res Intellect Disabil 2013;26:47-62.

17. Vereenooghe L, Langdon PE. Psychological therapies for people with intellectual disabilities: a systematic review and meta-analysis. Res Dev Disabil 2013;34:4085-102.

18. Deb S, Chaplin R, Sohanpal S, et al. The effectiveness of mood stabilizers and antiepileptic medication for the management of behaviour problems in adults with intellectual disability: a systematic review. J Intellect Disabil Res 2008;52(Pt 2):107-13.

19. Deb S, Sohanpal SK, Soni R, et al. The effectiveness of antipsychotic medication in the management of behaviour problems in adults with intellectual disabilities. J Intellect Disabil Res 2007;51:766-77.

20. Melville CA, Johnson PC, Smiley E, et al. Problem behaviours and symptom dimensions of psychiatric disorders in adults with intellectual disabilities: An exploratory and confirmatory factor analysis. Res Dev Disabil 2016;55:1-13. 
21. Moher D, Liberati A, Tetzlaff J, et al. Preferred reporting items for systematic reviews and meta-analyses: the PRISMA statement. PLoS Med 2009;6:e1000097.

22. American Psychiatric Association. Diagnostic and Statistical Manual of Mental Disorders. 4th edn. Wachington DC: American Psychiatric Publishing, 1994

23. Felce D, Kerr M, Hastings RP. A general practice-based study of the relationship between indicators of mental illness and challenging behaviour among adults with intellectual disabilities. J Intellect Disabil Res 2009;53:243-54.

24. American Psychiatric Association. Diagnostic and statistical manual of mental disorders. 3rd edn. Washington, DC: American Psychiatric Publishing, 1980

25. Petticrew M, Roberts H. Systematic reviews in the social sciences. $A$ practical guide. London: Blackwell Publishing, 2006.

26. Atkins $D$, Best $D$, Briss PA, et al. Grading quality of evidence and strength of recommendations. Br Med J 2004;328:1490.

27. Balshem $\mathrm{H}$, Helfand $\mathrm{M}$, Schünemann $\mathrm{HJ}$, et al. GRADE guidelines: 3. Rating the quality of evidence. J Clin Epidemiol 2011;64:401-6.

28. Guyatt GH, Oxman AD, Vist G, et al. GRADE guidelines: 4. Rating the quality of evidence--study limitations (risk of bias). J Clin Epidemiol 2011;64:407-15.

29. Singh J. Critical appraisal skills programme. Journal of Pharmacology and Pharmacotherapeutics 2013;4:76-7.

30. Critical Appraisal Skills Programme (CASP), 2014.

31. Horner RH, Carr EG, Halle J, et al. The use of single-subject research to identify evidence-based practice in special education. Except Child 2005;71:165-79.
32. Aman MG, White AJ, Vaithianathan C, et al. Preliminary study of imipramine in profoundly retarded residents. J Autism Dev Disord 1986;16:263-73.

33. Rosenquist PB, Bodfish JW, Thompson R. Tourette syndrome associated with mental retardation: a single-subject treatment study with haloperidol. Am J Ment Retard 1997;101:497-504.

34. Lindauer SE, DeLeon IG, Fisher WW. Decreasing signs of negative affect and correlated self-injury in an individual with mental retardation and mood disturbances. J Appl Behav Anal 1999;32:103-6.

35. White TJ, Aman MG. Pimozide treatment in disruptive severely retarded patients. Aust N Z J Psychiatry 1985;19:92-4

36. Zarkowska EC. A behavioural intervention for Gilles de la Tourette syndrome in a severely mentally handicapped girl. J Ment Defic Res 1981;1989:245-53.

37. Aman MG, Singh NN, Stewart AW, et al. The aberrant behavior checklist: a behavior rating scale for the assessment of treatment effects. Am J Ment Defic 1985;89:485-91.

38. National Insitute for Health and Care Excellence. Mental health problems in people with learning disabilities: prevention, assessment and management (NG54). NICE Guideline 54. 2016 nice.org.uk/ guidance/ng54.

39. Flynn S, Vereenooghe L, Hastings RP, et al. Measurement tools for mental health problems and mental well-being in people with severe or profound intellectual disabilities: A systematic review. Clin Psychol Rev 2017;57:32-44.

40. Checklists CASP. Oxford: CASP.

41. Slosson R. Slosson Intelligence Test for Children and Adults. East Aurora, New York: Slosson Educational Publications, 1975. 